\title{
In Search of Multi-Peaked Reflective Spectrum with Optic Fiber Bragg Grating Sensor for Dynamic Strain Measurement
}

\author{
H. Tai \\ Langley Research Center, NASA \\ Hampton VA 23681
}

\begin{abstract}
In a typical optic fiber Bragg grating (FBG) strain measurement, unless in an ideal static laboratory environment, the presence of vibration or often disturbance always exists, which often creates spurious multiple peaks in the reflected spectrum, resulting in a non-unique determination of strain value. In this report we attempt to investigate the origin of this phenomenon by physical arguments and simple numerical simulation. We postulate that the fiber gratings execute small amplitude transverse vibrations changing the optical path in which the reflected light traverses slightly and non-uniformly. Ultimately, this causes the multi-peak reflected spectrum.
\end{abstract}

\section{Introduction}

In recent years, the use of optical fiber Bragg grating sensors has gained popularity for sensing strain, temperature, and pressure etc., especially for large structures, such as aircraft wings and bodies in real time. FBG sensors offer several inherent advantages over conventional electrical resistance foil strain gauges. They are immune to electromagnetic interference, and are light weight, portable, and self-referencing and up to several hundred sensors can be incorporated along a single fiber. Despite these advantages, the development of Bragg grating sensors as a mainstream strain sensing technique, especially in a real dynamic environment, has been hampered by a number of factors, such as lack of conclusive information about sensor reliability and repeatability. Sometimes the reflected signal does not give a unique value; the reflected peak tends to spilt and the strain value has to be guessed. In this report we would like to investigate this issue using physical principles and numerical simulation, and hopefully we can shed some light on this topic.

\section{Contributing factors of spectrum signal noise}

Consider a scenario of Bragg grating fiber sensor imbedded in the skin of a morphing wing of an airplane during flight, where the measured strain values are sought to control the shape of the wing. There are many factors that could affect the measured strain value such as engine noise, wind and air stream noise, engine vibration (higher frequency), flutter (much lower frequency) noise and airplane natural vibration. Among all the possible input noises, we believe the most dominant ones are the engine vibration and air stream induced vibrations which generate a much higher frequency and amplitude. These vibrations, in our belief, ultimately ruin the precision of measured strain values.

\section{Treating the fiber as part of an elastic continuum mechanically}

Since the optical Bragg grating fiber is embedded in the skin of a structure, and due to its small mass, we assign all its mechanical properties, such as stiffness and modulus of the fiber to those of the host, for example the aluminum structure as far as mechanical response 
is concerned. Fortunately the material properties are very close to the optical fiber's ${ }^{1}$. The optical fiber retains only its optical property. In an elastic medium a small applied shear force produces a true static configuration that returns to the original un-deformed state when the force is removed. The elastic equation of motion is often written in vector form ${ }^{2}$. $\rho \frac{\partial^{2} \mathbf{u}}{\partial t^{2}}=\mu \nabla^{2} \mathbf{u}+\left(K+\frac{1}{3} \mu\right) \nabla(\nabla \square \mathbf{u})+\rho \mathbf{f}$

Where $\rho$ is the density, mass/volume; $\mu$ is known as the shear modulus; $K$ is the bulk modulus, both have the dimension of force per unit area. $\mathbf{f}$ is an external driving force. We separate the general displacement field $\mathbf{u}$ into its longitudinal and transverse components,

$\mathbf{u}=\mathbf{u}_{l}+\mathbf{u}_{t}$

Where $\nabla \times \mathbf{u}_{l}=0, \quad \nabla\left\lceil\mathbf{u}_{t}=0\right.$

We further can separate longitudinal and transverse components into two independent equations

$\left.\rho \frac{\partial^{2} \mathbf{u}_{l}}{\partial t^{2}}-\left(K+\frac{4}{3} \mu\right) \nabla^{2} \mathbf{u}_{l}\right)=\rho \mathbf{f}_{l}$

and

$\rho \frac{\partial^{2} \mathbf{u}_{t}}{\partial t^{2}}-\mu \nabla^{2} \mathbf{u}_{t}=\rho \mathbf{f}_{t}$

In the absence of external forces, either component satisfies a simple homogeneous wave equation

$\frac{1}{v_{l}^{2}} \frac{\partial^{2} \mathbf{u}_{l}}{\partial t^{2}}=\nabla^{2} \mathbf{u}_{l}$

where $v_{l}=\left(\frac{K+\frac{4}{3} \mu}{\rho}\right)^{1 / 2}$

and

$\frac{1}{v_{t}^{2}} \frac{\partial^{2} \mathbf{u}_{t}}{\partial t^{2}}=\nabla^{2} \mathbf{u}_{t}$

where $v_{t}=\left(\frac{\mu}{\rho}\right)^{1 / 2}$ 
Note that from the above equations, the speed, $v_{l}>v_{t}$, implying that an elastic medium offers greater resistance to a longitudinal disturbance than to a transverse one. By the same argument, one of the transverse components parallel to the surface can also be neglected. Therefore, we only have to deal with the transverse component denoted as $u_{\perp}$ which is perpendicular to the structure surface. Furthermore, we assume the fiber grating is parallel to the wave propagation direction which lies along the $\mathrm{x}$ axis.

We rewrite Eq. (5) and replace $v_{t}$ by $v$

$\frac{1}{v^{2}} \frac{\partial^{2} u_{\perp}}{\partial t^{2}}-\frac{\partial^{2} u_{\perp}}{\partial x^{2}}=f_{t}$

The right hand side provides a traveling wave solution of much lower frequency and is ignored here.

For simplicity, let us assume the driving force is located at $\mathrm{x}=0$ with simple harmonics form

$f_{t}=F_{0} e^{-i \omega t}$

Then, following ${ }^{3}$, we obtain the particular solution of Eq. (7) as

$u_{\perp}=\frac{1}{-i \omega} \frac{F_{0}}{\rho v} e^{i k(x-v t)}=\frac{1}{\omega} \frac{F_{0}}{\rho v} e^{i k(x-v t+\pi / 2)}$

where the wave vector $k$ is

$k=\frac{\omega}{v}=\frac{2 \pi}{\Omega}$

From Eq. (9) we realize that for high frequency vibration, we can achieve a $\Omega$ that is comparable to our fiber grating length.

\section{A stretched fiber caused by the vibration}

As shown in Figs. 1a and 1b, we depict a fiber lying along the $\mathrm{x}$-axis when there is no vibration, but as an external disturbance such as vibration strikes, the host medium along with the fiber reacts as an elastic continuum in a wave motion. We have shown here is that for an ideal case without losing generality the total grating length is one quarter $(1 / 4)$ of the vibration wavelength $\Omega$. We assume that the fiber is executing a harmonic motion represented thus

$y(x)=A \sin (k x+\phi)$

Where the harmonic time dependence is omitted; $A$ is the amplitude and $\phi$ is a time independent phase angle. In other words, Eq. (10) represents the wave motion at instant time $t$, as if we take a snap shot at time $t$ as the wave is passing by. Or we can argue that a 
standing wave is created and that the time and space variables are separated and time dependence is absorbed by the amplitude ${ }^{4}$.

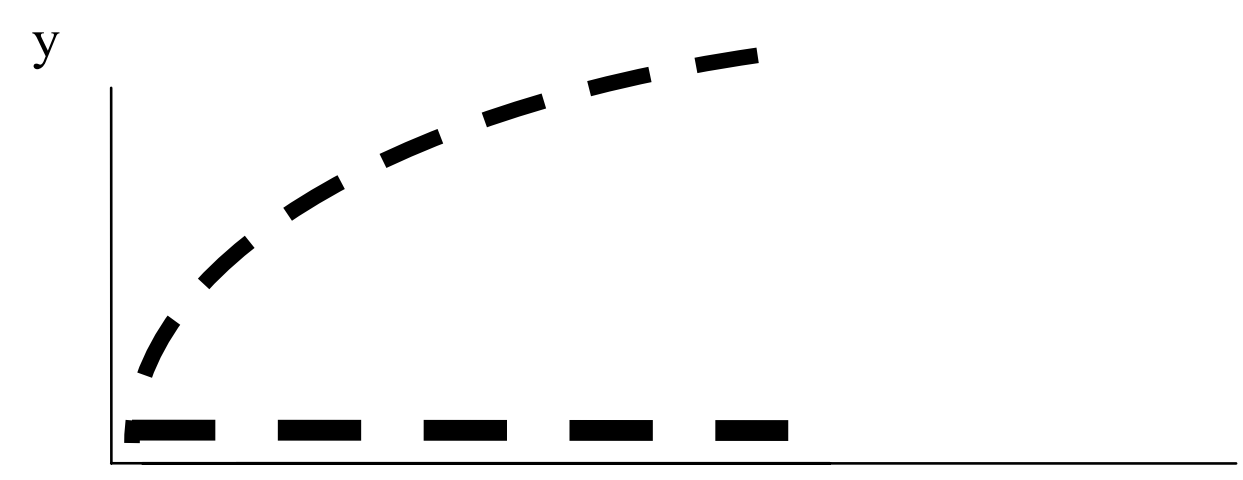

X

Fig. 1a the total Grating length happens to be approximately $1 / 4$ of vibration length with phase angle of $0^{0}$

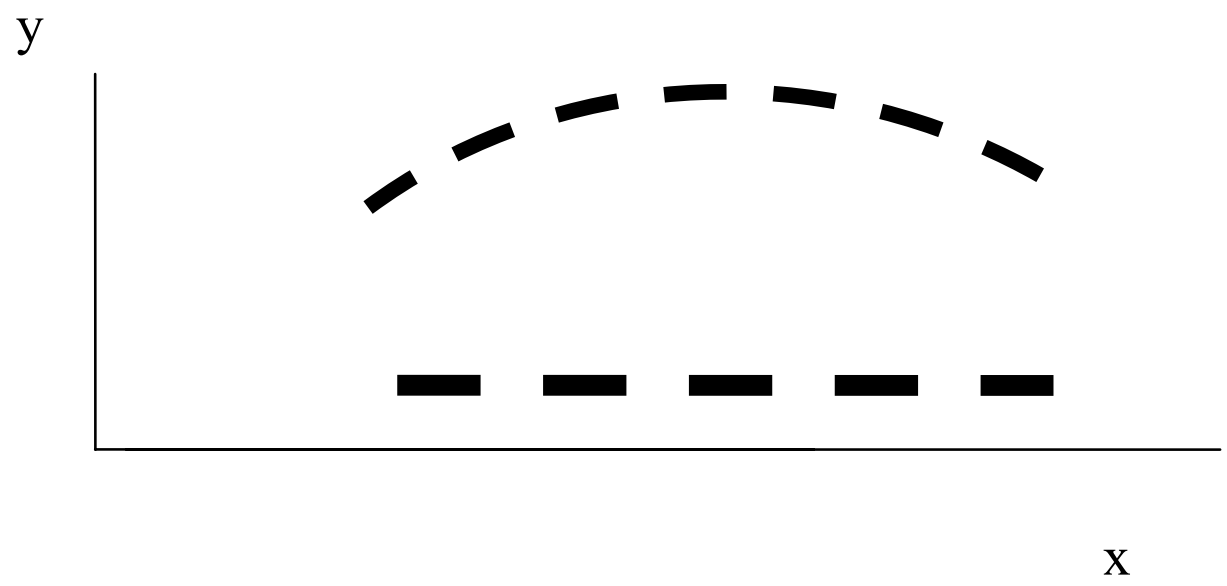

Fig. $1 \mathrm{~b}$ the total Grating length is $1 / 4$ of vibration wavelength with phase angle $45^{0}$ 
In Figs. 1a \& 1b, the total sensor grating length is equal to one quarter of the vibrating wavelength, but with different phase angles. Of course, in general, the total grating length can be any fraction of the vibration wavelength depending on time, wavelength, speed etc.

We consider a fiber grating length of $L$ (from $x=0$ to $x=L$ ), which consists of alternating sectional length of $l$ and $r$, each having an index of refraction of $n$ and $n^{\prime}\left(n^{\prime}=n+\Delta n\right)$. To reach maximum reflectivity (tuned), the following conditions have to be satisfied ${ }^{5}$ :

$r=\lambda_{0} / 4 n^{\prime}, \quad l=\lambda_{0} / 4 n$

And the grating period $d$

$d=r+l=\frac{\lambda_{0}}{4}\left(\frac{1}{n^{\prime}}+\frac{1}{n}\right)=\frac{\lambda_{0}}{2 n_{\text {eff }}}$

where $n_{\text {eff }}=\frac{2 n^{\prime} n}{n+n^{\prime}}$

And $\lambda_{0}$ is the "tuned" optical wavelength.

In the static case the length $r$ and $l$ are constants, but in a stretched case under vibration, both $r$ and $l$ become a function of $x$ stretched to $r$ ' and $l$ ' and are measured along the arc. If a simple sine wave is adopted and the sensor length is approximately equal to $1 / 4$ vibration period $\Omega$, then the vertical displacement can be expressed as

$y=A \sin (\pi x / 2 \Omega+\phi)$, then

$d y / d x=\frac{A \pi}{2 \Omega} \cos (\pi x / 2 \Omega+\phi)$

$r^{\prime}=\sqrt{1+(d y / d x)^{2}} r$

Likewise, the value $l$ changes to $l$ ' and $\phi$ is the time independent phase angle.

This assumption forms the theoretical base of our model. The computation was carried out using standard transfer matrix method which we have reported earlier. ${ }^{6,7}$

\section{Simulation Results}

There are two parameters that characterize the vibration; frequency (wavelength) and amplitude. In order to show qualitatively the reflective spectrum with respect to amplitude, we let the sensor length approximately equal one quarter of the vibration period $\Omega$, for vibration amplitude $A=1 . e+4,1 . e+5,5 . e+5$ and $1 . \mathrm{e}+6 \mathrm{~nm}$ and for initial phase angle $\phi=0$ and $\pi / 4$ respectively. It is obvious that for different phase angles, the sensor length occupies different segments of the wave period resulting in a different distribution of grating distance change. Intuitively, we knew that a sine curve of small argument at phase angle $\phi=0$, behaves linearly. In other words, the grating planes on the left (trailing edge)get stretched linearly. While for phase angle $\phi=\pi / 4$, the central part of the grating planes get stretched less but more uniformly (keep in mind that the change of grating distance depends upon the slope). The different distributions of grating plane change results in a 
totally different reflective spectra, shown in figs. $2 \mathrm{c}, 2 \mathrm{~d}, 2 \mathrm{~g}$ and $2 \mathrm{~h}$. However when the amplitude is small, the reflective spectrum hardly show any abnormality, but instead exhibits a perfect, distinct, single peak spectrum governed by the Bragg law. In all the simulation results, we have assumed the sensor length $L=3 \mathrm{~mm}$ and the speed $u_{\perp}=3000$ $\mathrm{m} / \mathrm{s}$ which translates to the forced vibration frequency $\omega / 2 \pi$, of about $250 \mathrm{~K}$ cps, which is in the ultrasound frequency range. In our analysis, we have not included the damping and photo-elastic effects ${ }^{6}$.

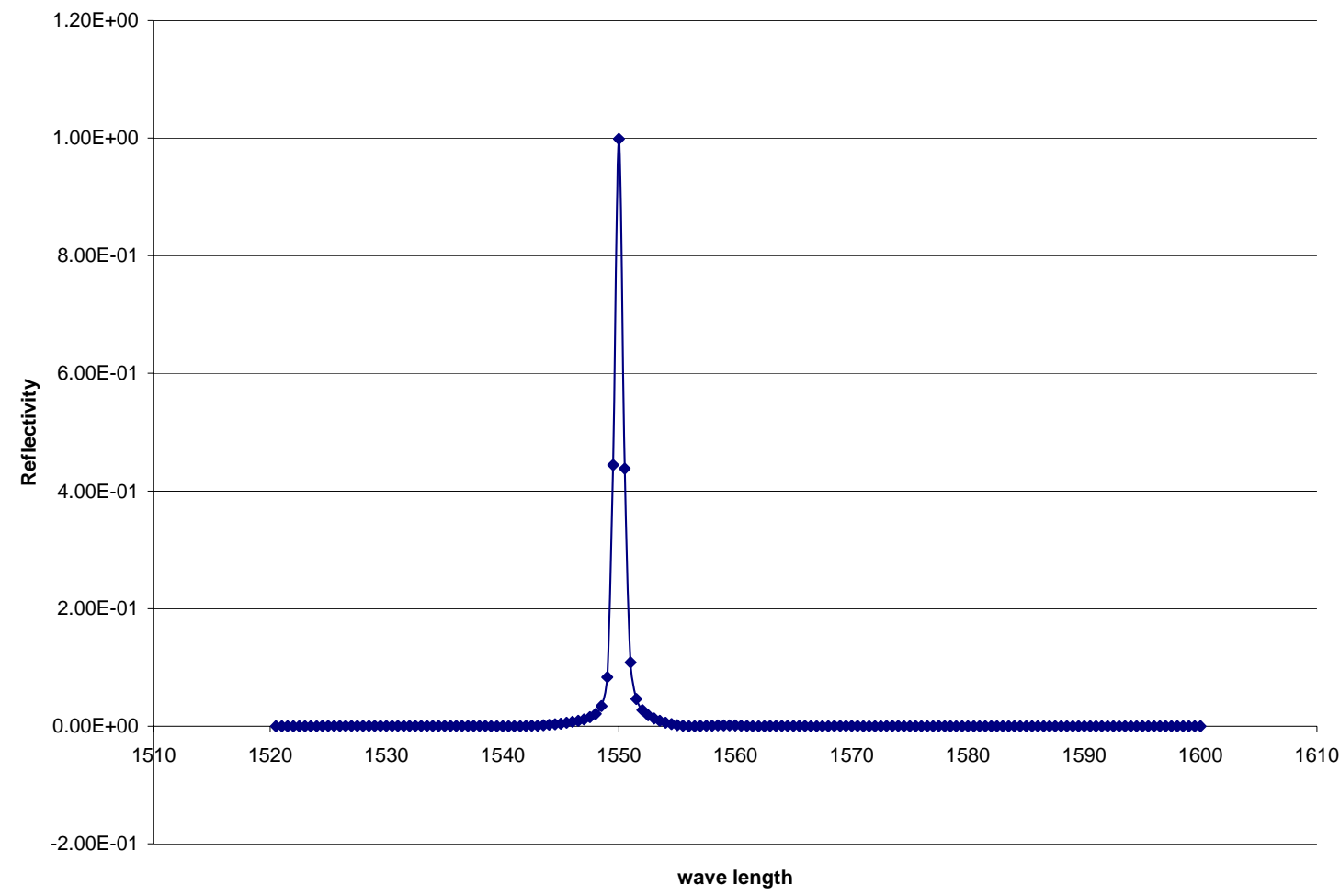

Fig. 2a Reflectivity versus wavelength for vibration amplitude $=1 . \mathrm{e}+4 \mathrm{~nm}$ with phase $=0^{0}$ 


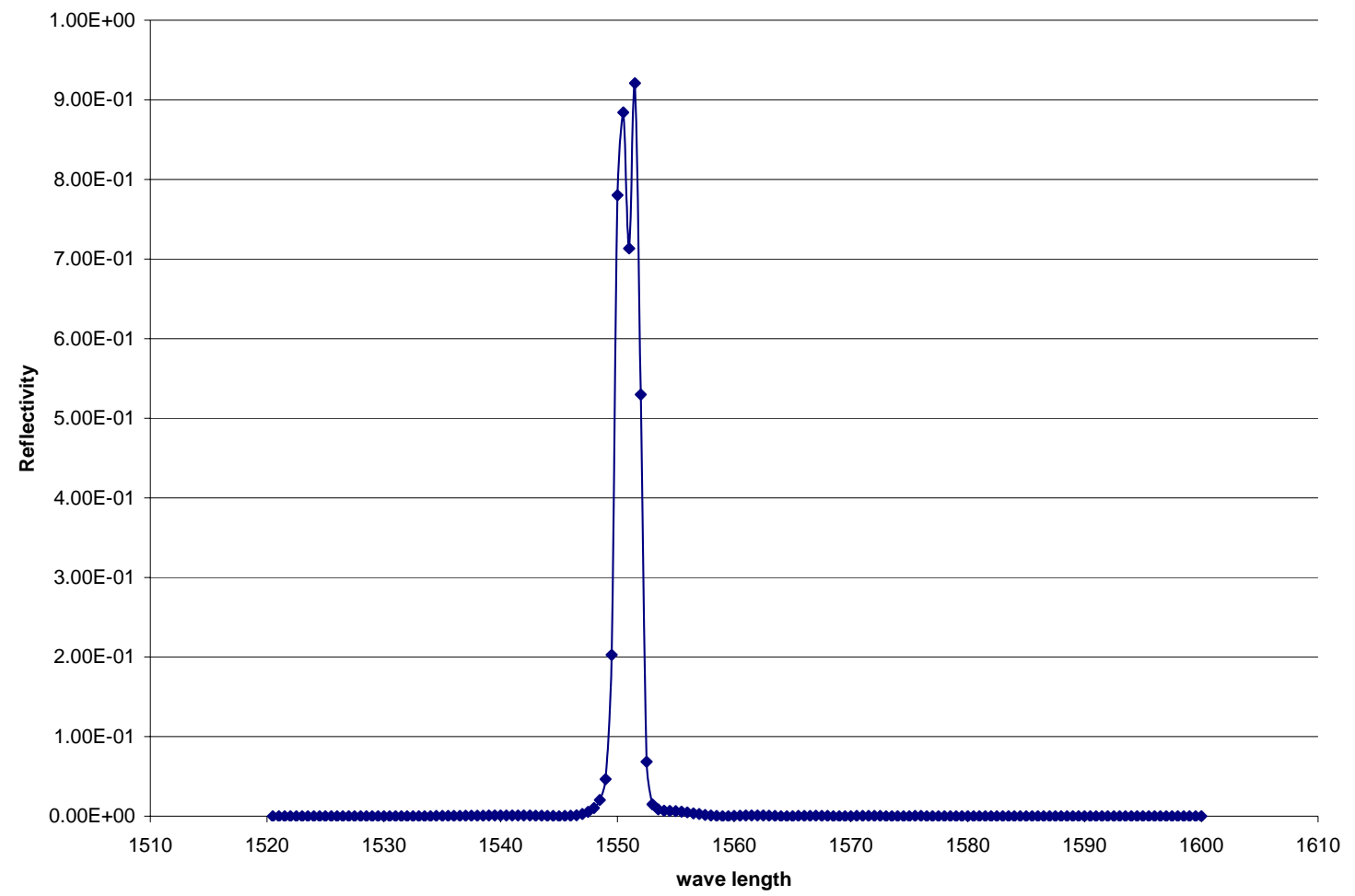

Fig. 2b Reflectivity versus wavelength for vibration amplitude $=1 . \mathrm{e}+5 \mathrm{~nm}$ with phase $=0^{0}$

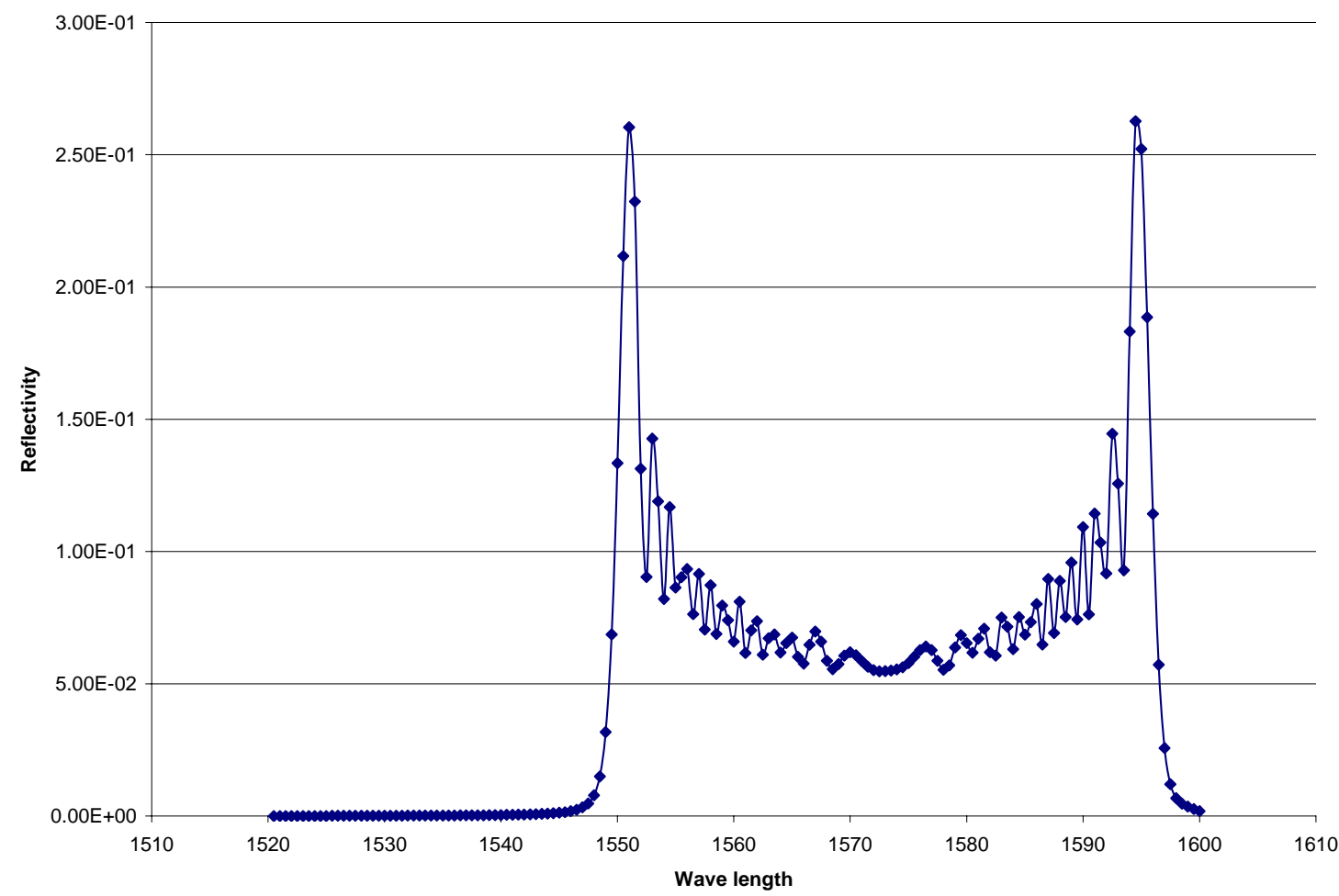

Fig. $2 \mathrm{c}$ Reflectivity versus wavelength for vibration amplitude $=5 . \mathrm{e}+5 \mathrm{~nm}$ with phase $=0^{0}$ 


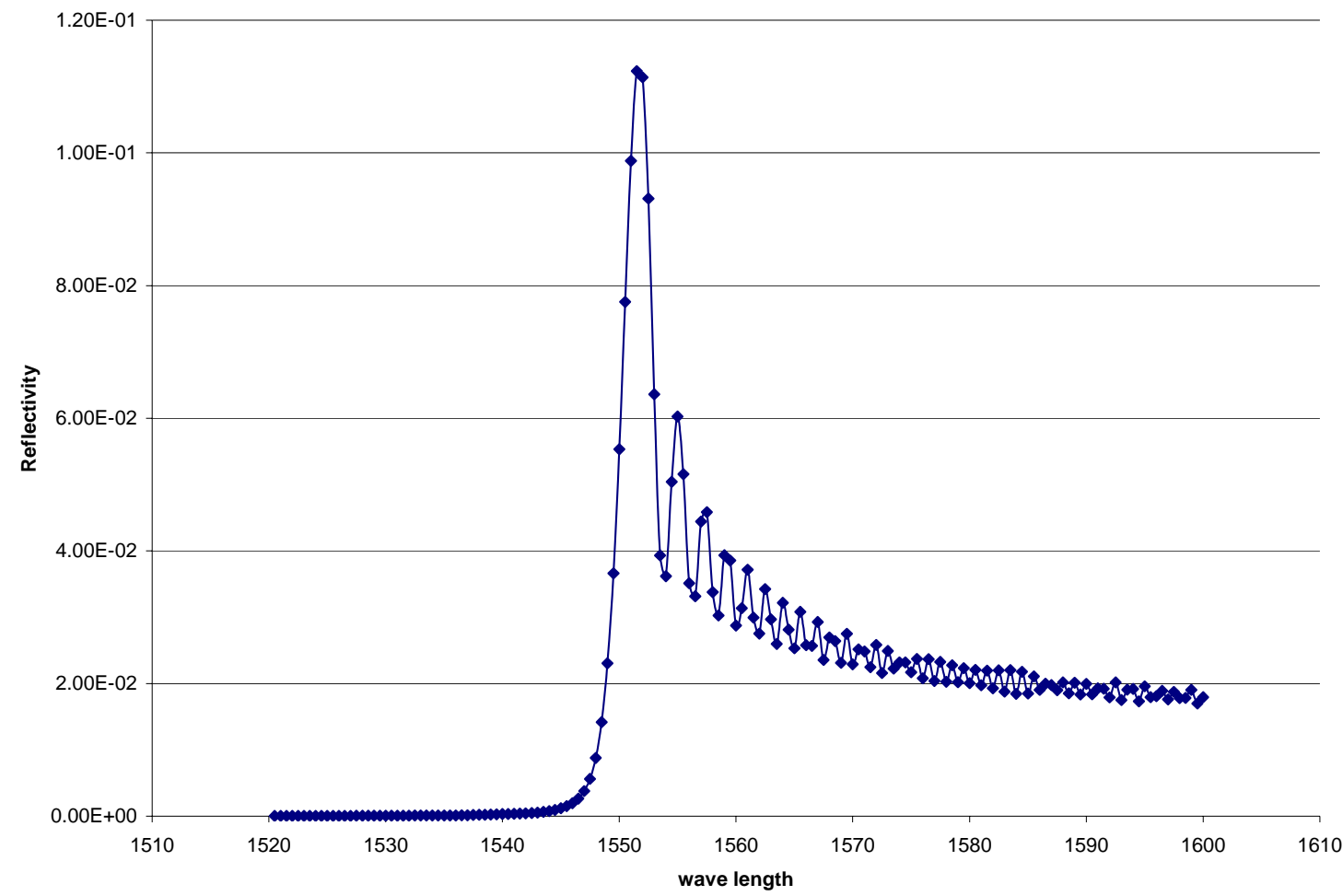

Fig. $2 \mathrm{~d}$ Reflectivity versus wavelength for vibration amplitude $=1 . \mathrm{e}+6 \mathrm{~nm}$ with phase $=0^{0}$

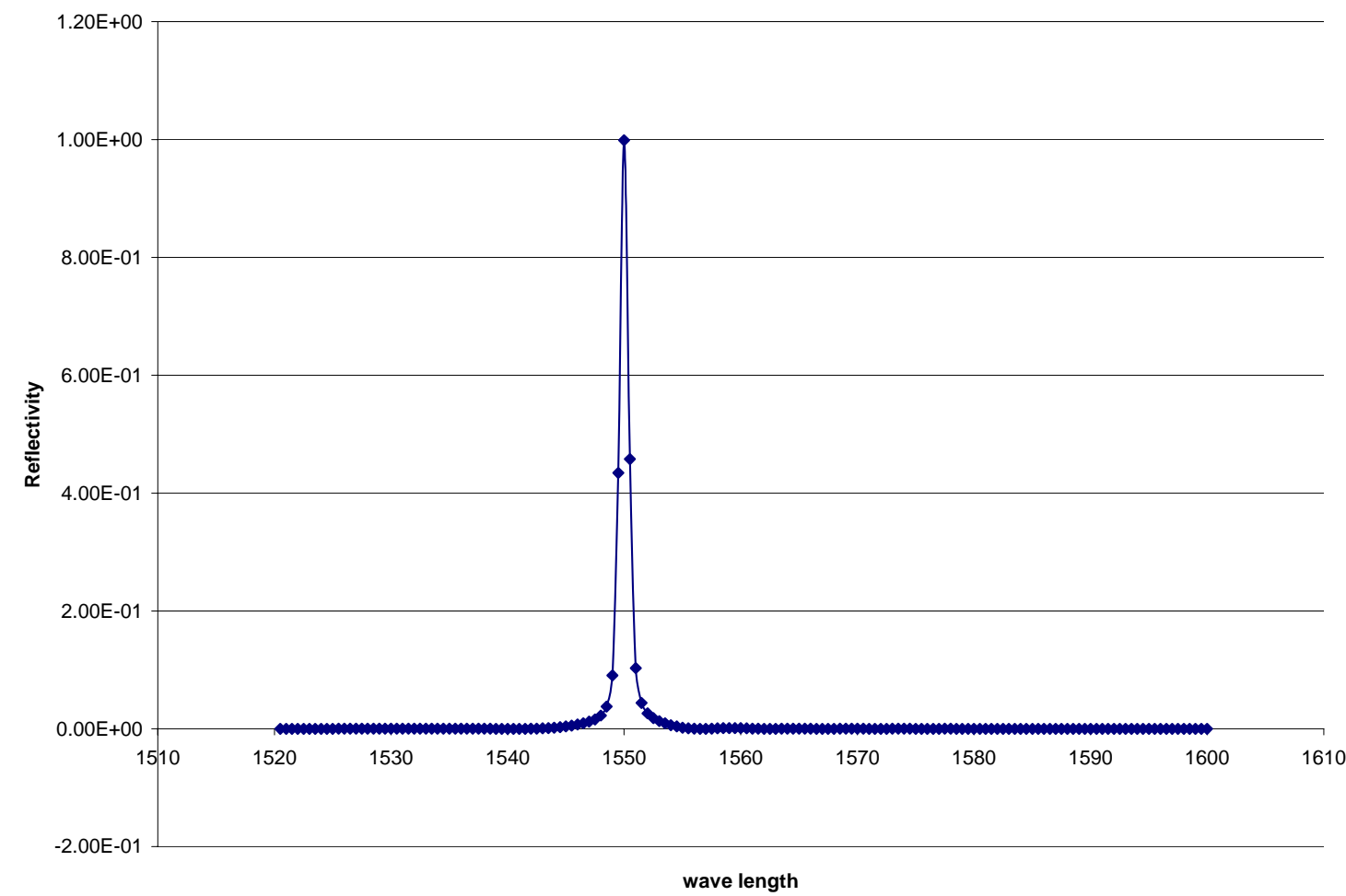

. 2e Reflectivity versus wavelength for vibration amplitude $=1 . \mathrm{e}+4 \mathrm{~nm}$ with phase $=45^{0}$ 


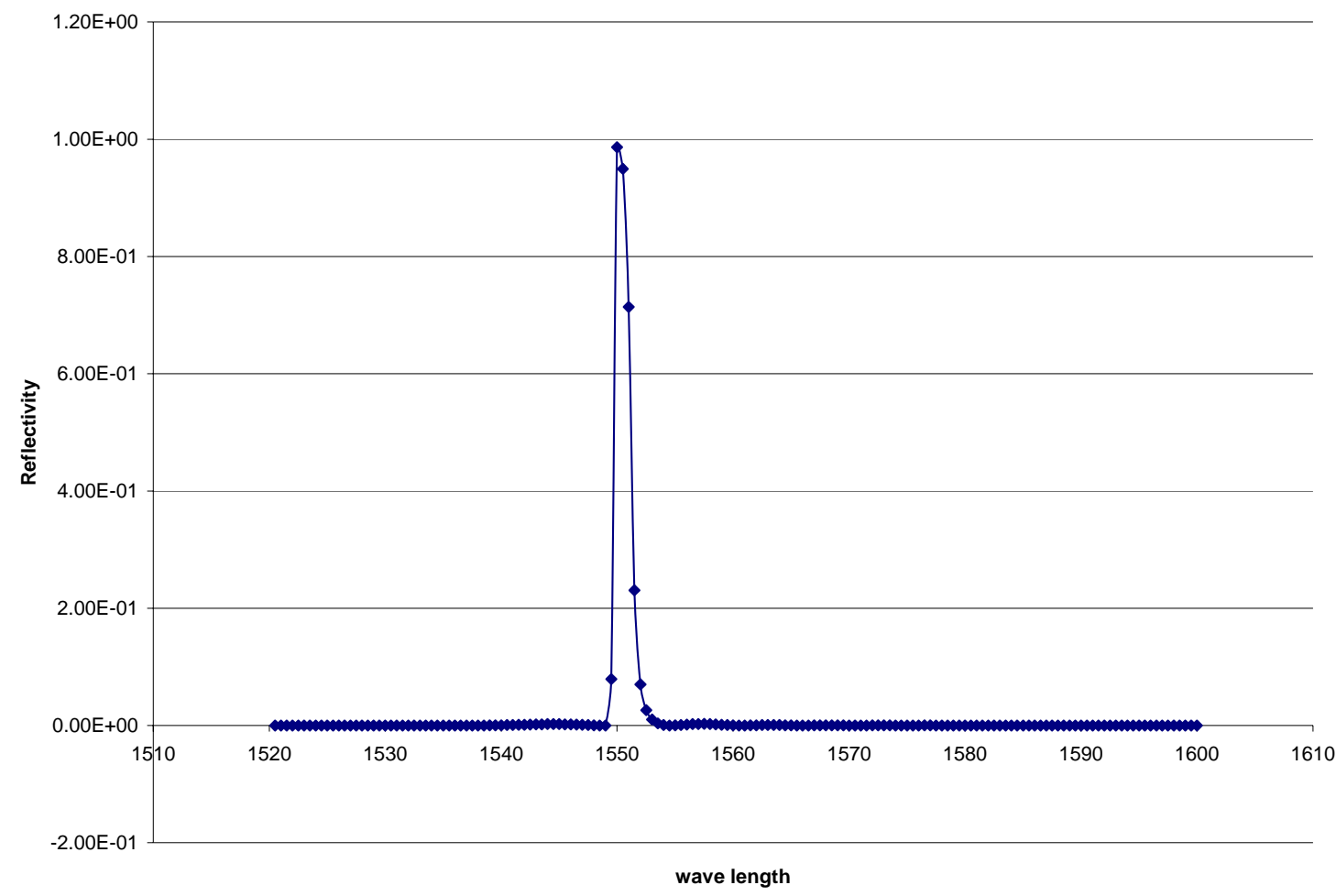

$2 \mathrm{f}$ Reflectivity versus wavelength for vibration amplitude $=1 \mathrm{e}+5 \mathrm{~nm}$ with phase $=45^{0}$

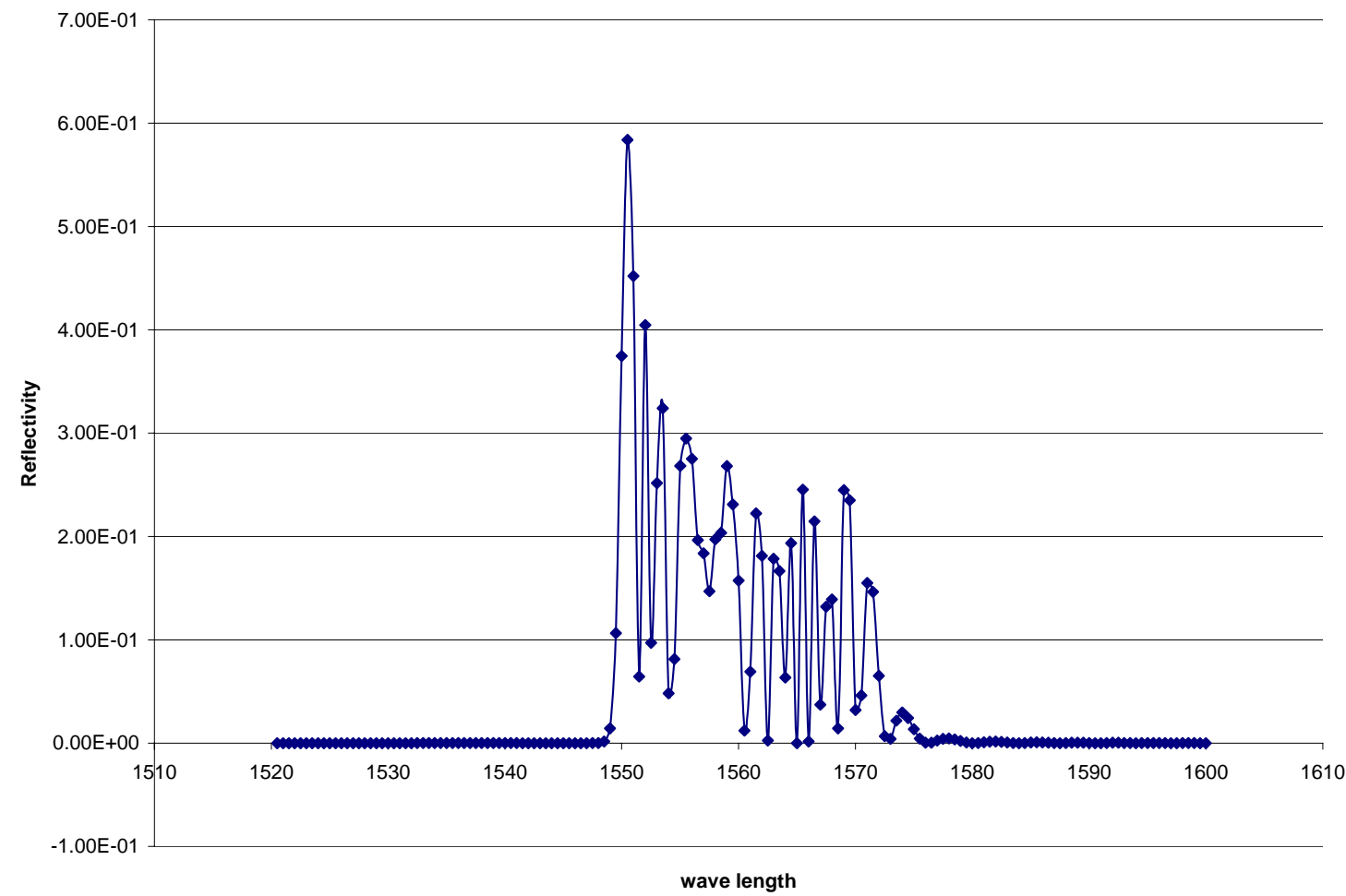

$2 \mathrm{~g}$ Reflectivity versus wavelength for vibration amplitude $=5 . \mathrm{e}+5 \mathrm{~nm}$ with phase $=45^{0}$ 


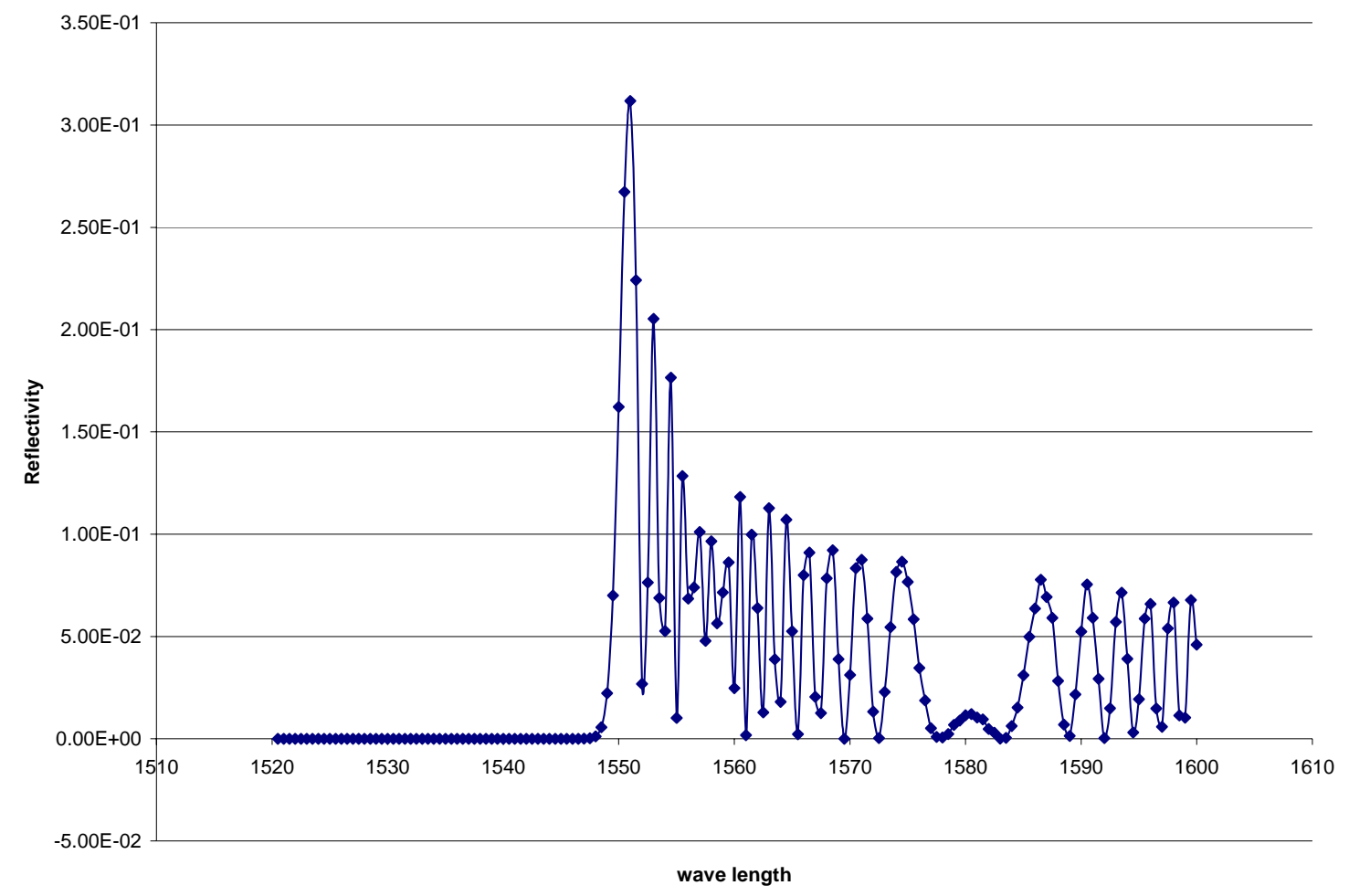

$2 \mathrm{~h}$ Reflectivity versus wavelength for vibration amplitude $=1 . \mathrm{e}+6 \mathrm{~nm}$ with phase $=45^{0}$

\section{Conclusion and Discussion}

We have gained some understanding with respect to dynamic strain measurement that, indeed, vibration or any disturbance may cause non-unique strain determination. We argued physically that the dominant mode of the vibration that interferes and causes our erroneous strain measurement is the transverse (out of surface) component of the external vibration source. For a given frequency (in our case at $250 \mathrm{~K} \mathrm{cps}$ ), depending upon the amplitude, if the amplitude is small, e.g., $A=1 . e+4 \mathrm{~nm}$ which is $10^{-3} \mathrm{~cm}$, we can uniquely determine the strain values. However, when the amplitude becomes larger, say $>5.10^{-2} \mathrm{~cm}$, the reflective spectrum will show split peaks. Intuitively, a local disturbance of amplitude of $5.10^{-2} \mathrm{~cm}$ is highly possible. The reflective spectrum pattern can qualitatively and uniquely be correlated to the distribution of grating plane changes. As far as the frequency is concerned, lower frequency means longer wavelength $\Omega$. For the same amplitude, the slope of the sine curve is less steep; therefore the grating plane distance changes less, resulting in weaker split peaks and vice versa. For all the analyses and calculations reported in the literature, a time independent approach is always assumed, that is at any time the measurement should yield the same results. Obviously this is not the case here. At time instant $t$ and the reflective spectrum obtained which is time dependent unless the amplitude is small, in which case the reflective spectra are almost identical. We also assume there is no time delay in the laboratory equipment and the speed of light is infinite. In reality, of course, this is not quite true. When the frequency becomes higher, the time constant (period) gets smaller to a point that falls into the range of delay time of the 
measuring equipment. In such a scenario, the situation is much tougher to handle. Another interesting observation seen by examining Fig. $2 \mathrm{a}$ to $2 \mathrm{~h}$, suggests that this analysis may provide a highly sensitive sensor for shape sensing, i.e., a lateral movement on the order of $10^{-2} \mathrm{~cm}$ can be detected. What was shown here is the unstrained case, but for the strained case we need to superimpose these two effects. However, the present analysis still stands. We conclude by stating that the fiber optic Bragg sensor works on the principle of constructive reflection of light on a fixed distance of the grating plane. Any mechanical disturbance that changes the distance between the reflecting planes non-uniformly that will result in some degree of destructive interference, which is manifested in the spectrum.

\section{References}

1. David R. Lide, Editor in Chief, "CRC handbook of Chemistry and Physics", $74^{\text {th }}$ Ed., 1993

2. Alexander L. Fetter and John Dirk Walecka, "Theoretical Mechanics of Particles and Continua", McGraw-Hill Book company, Inc., 1980

3. Philip M. Morse. "Vibration and Sound", $2^{\text {nd }}$ ed., McGraw-Hill Book company, Inc., 1948

4. Robert Resnick and David Halliday, "Physics", John Wiley \& Sons, Inc., 1978

5. H. Tai, "Theory of Fiber Optical Bragg Grating-Revisited", SPIE's 48Annual Meeting , August 6-7, 2003

6. H. Tai, "Simple numerical simulation of strain measurement", SPIE's 47Annual Meeting, July 5-11, 2002.

7. A. K. Ghatak and K. Thyagarajan, “Optical Electronics”,Cambridge Univ. Press, 1989 